Article

\title{
Isolation, Structural Elucidation of Three New Triterpenoids from the Stems and Leaves of Schisandra chinensis (Turcz) Baill.
}

\author{
Feng Qiu, Han Liu, Huan Duan, Pian Chen, Shao-Juan Lu, Guang-Zhong Yang * and \\ Xin-Xiang Lei * (1D
}

School of Pharmaceutical Sciences, South-Central University for Nationalities, Wuhan 430074, China; 2016110449@mail.scuec.edu.cn (F.Q.); 2017110424@mail.scuec.edu.cn (H.L.); 2016110444@mail.scuec.edu.cn (H.D.); chenpian1104@126.com (P.C.); 201621154052@mail.scuec.edu.cn (S.-J.L.)

* Correspondence: yanggz@mail.scuec.edu.cn (G.-Z.Y.); xxlei@mail.scuec.edu.cn (X.-X.L.); Tel.: +86-27-67841196 (X.-X.L.)

Received: 13 June 2018; Accepted: 27 June 2018; Published: 4 July 2018

\begin{abstract}
Schisandra chinensis (Turcz) Baill. is sufficiently well known as a medicinal plant worldwide, which modern research shows has many pharmacological activities such as hepatoprotective, anti-inflammatory effect, potent anti-HIV-1 activity, anti-tumor effect, and activity on the central nervous system. With considerable chemical investigation, three new triterpenoids (1-3), together with four known triterpenoids were isolated from the S. chinensis (Turcz) Baill. Their structures were elucidated by 1D- and 2D-NMR spectroscopic analyses, single-crystal X-ray diffraction and high-resolution mass spectroscopy, which were identified as Schisanlactone I (1), Schinalactone D, (2), Schisanlactone J, (3) Kadsuphilactone B (4), Schisanlactone C (5), Schisphendilactone B (6), and Schinchinenlactone A (7). The cytotoxicity of those compounds (1-7) was tested against Hep-G2 cell lines, but no apparent antitumor activity was observed at $50 \mu \mathrm{g} / \mathrm{mL}$ using MTT method.
\end{abstract}

Keywords: Schisandra chinensis (Turcz) Baill.; triterpenoids; cytotoxicity

\section{Introduction}

Schisandra chinensis (Turcz) Baill., Chinese magnolia vine, is widely distributed in China, the most eastern parts of Russia, Japan, Korea, USA, Europe, and all over the world [1]. The plant of S. chinensis (Turcz) Baill. can be found in the Chinese Pharmacopoeia, Russian Pharmacopoeia, Japanese Pharmacopoeia, Korean Pharmacopoeia, American Pharmacopoeia, and the International Pharmacopoeia [2]. According to various authors, the genus Schisandra includes from 20 to 30 species, and the major chemical composition of S. chinensis (Turcz) Baill. is lignans and triterpenes [2,3]. The seeds and fruits have been used to treat various diseases such as cough insomnia and arthritis [4]. Modern research shows it has many pharmacological activities such as hepatoprotective, anti-inflammatory effect, potent anti-HIV-1 activity, anti-tumor effect and activity on the central nervous system [5-9]. Most of phytochemical studies have concentrated on the analysis of fruits, and a great majority of them investigated lignans, but our studies of vegetative parts of stems and leaves are scarce. As a part of our effort to search for novel triterpene from S. chinensis (Turcz) Baill., we report here the isolation and structure determination of the new triterpenoids: Schisanlactone I (1), Schinalactone D (2), and Schisanlactone J (3), in addition to four known triterpenoids: Kadsuphilactone B (4), Schisanlactone C (5), Schisphendilactone B (6) and Schinchinenlactone A (7) (Figure 1). 


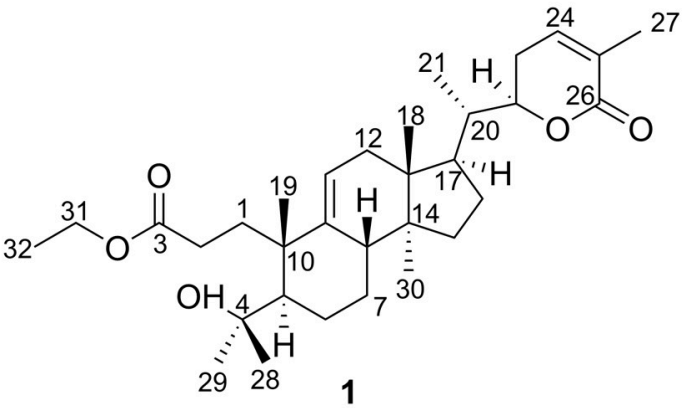

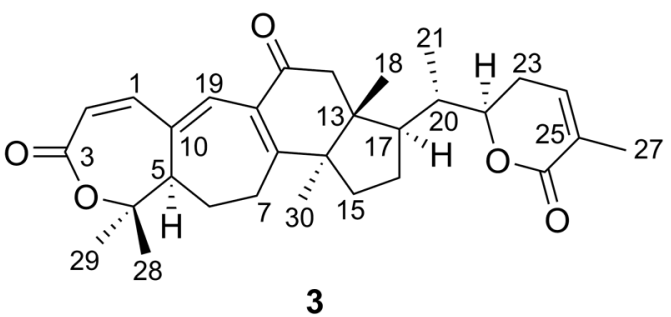<smiles></smiles>

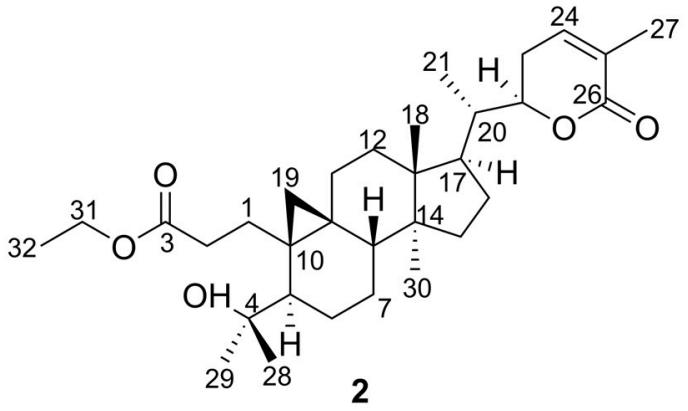



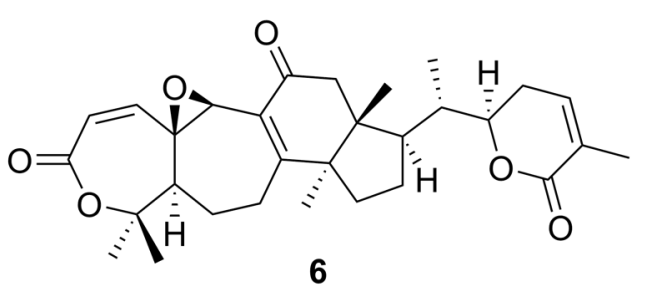<smiles></smiles>

Figure 1. Chemical structures of triterpenoids 1-7.

\section{Results and Discussion}

\section{Structure Elucidation}

The structures of the new compounds (1-3) were elucidated on the basis of extensive spectroscopic analyses, including a series of 1D- and 2D-NMR experiments $\left({ }^{1} \mathrm{H}^{-1} \mathrm{H}\right.$ COSY, HSQC and HMBC), single-crystal $X$-ray diffraction, IR spectrum, and mass spectrometry data. The known compounds (4-7) were identified by comparison of their experimental spectral data with the literature data [10-12].

Compound 1 was obtained as white amorphous powder with the molecular formula determined to be $\mathrm{C}_{32} \mathrm{H}_{50} \mathrm{O}_{5}$ according to the molecular ion peak $[\mathrm{M}+\mathrm{H}]^{+}$at $m / z 515.3737$ (calculated for 515.3758) observed in HR-ESI-MS (Figure S9) and NMR spectroscopic data (Figures S1-S6). Analysis of NMR data (Table 1) indicated that compound 1 highly resembled Schisanlactone $H$ [13]. The only difference was the replacement of the ethoxycarbonyl group in 1, which could be deduced by the HMBC correlations (Figure S7) between $\mathrm{H}-31\left(\delta_{\mathrm{H}} 4.11, \mathrm{q}, J=7.13 \mathrm{~Hz}\right)$ and methyl group $\left(\delta_{\mathrm{C}} 14.57\right)$, ester group $\left(\delta_{\mathrm{C}} 177.05\right)$, otherwise ${ }^{1} \mathrm{H}-{ }^{1} \mathrm{H}$ COSY correlations (Figure S7) of H-31 $\left(\delta_{\mathrm{H}} 4.11\right)$ with H-32 $\left(\delta_{\mathrm{H}} 1.25\right)$. The absolute configuration (Figure 2$)$ of 1 was determined to be $(5 R, 8 S, 10 S, 13 R, 14 S, 17 R, 20 S, 22 R)$ 
by X-ray diffraction (Figure S8) using $\mathrm{Cu} \mathrm{K} \alpha$ radiation with a Flack parameter of 0.04 (9). Thus, the structure of compound 1 was identified as Schisanlactone I (Figure 1).

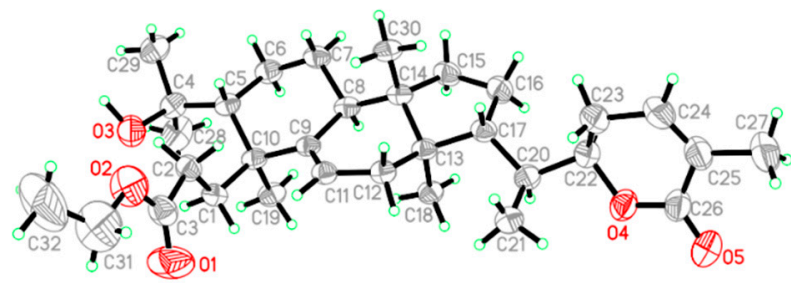

Figure 2. X-ray SAINT drawing of compound 1.

Compound 2 was obtained as white amorphous powder with the molecular formula determined to be $\mathrm{C}_{32} \mathrm{H}_{50} \mathrm{O}_{5}$ on the basis of the molecular ion peak $[\mathrm{M}+\mathrm{Na}]^{+}$at $\mathrm{m} / z 537.3551$ (calculated for 537.3556) observed in HR-ESI-MS (Figure S18) and NMR spectroscopic data (Figures S11-S16). Analysis of its NMR data (Table 1) indicated that compound 2 highly resembled Schinalactone C [14]. The only difference was the replacement of the ethoxycarbonyl group in 2, which could be deduced by the HMBC correlations (Figure S17) between H-31 $\left(\delta_{\mathrm{H}} 4.08, \mathrm{q}, J=7.12 \mathrm{~Hz}\right)$, methyl group $\left(\delta_{\mathrm{C}} 14.58\right)$, and ester carbonyl group $\left(\delta_{\mathrm{C}}\right.$ 176.31); otherwise, ${ }^{1} \mathrm{H}-{ }^{1} \mathrm{H}$ COSY correlates (Figure S17) H-31 $\left(\delta_{\mathrm{H}} 4.08\right)$ with H-32 $\left(\delta_{\mathrm{H}}\right.$ 1.22). Thus, the structure of compound 2 was identified as Schinalactone D (Figure 1).

Compound 3 was obtained as white amorphous powder with the molecular formula determined to be $\mathrm{C}_{30} \mathrm{H}_{38} \mathrm{O}_{5}$ on the basis of the molecular ion peak $[\mathrm{M}+\mathrm{H}]^{+}$at $\mathrm{m} / z 479.2790$ (calculated for 479.2798) observed in HR-ESI-MS (Figure S26) and NMR spectroscopic data (Figures S20-S25). Analysis of its NMR data (Table 1) indicated that compound 3 highly resembled Lancilactone A [15]. The main difference was the carbonyl carbon $(\mathrm{C}-11)$ in 3 and the hydroxyl replacement group of C-6 in Lancilactone A. In the HMBC spectrum (Figure 3), the correlations between $\mathrm{H}-19\left(\delta_{\mathrm{H}} 6.72\right)$ and the carbonyl group $\left(\delta_{\mathrm{C}} 199.85\right)$, between $\mathrm{H}-12\left(\delta_{\mathrm{H}} 2.52\right.$ and 2.83$)$ and the carbonyl group $\left(\delta_{\mathrm{C}} 199.85\right)$ revealed the carbonyl group was located at C-11, meanwhile C-6 $\left(\delta_{\mathrm{C}} 30.73\right)$ of 3 has no hydroxyl replacement group. So, the structure of compound 3 was identified as Schisanlactone J (Figure 1).



Figure 3. Key HMBC correlations and ${ }^{1} \mathrm{H}_{-}{ }^{1} \mathrm{H}$ COSY correlations.

Table 1. ${ }^{1} \mathrm{H}$ and ${ }^{13} \mathrm{C}-\mathrm{NMR}$ data (600 and $150 \mathrm{MHz}$, respectively, 1-3 in $\mathrm{CD}_{3} \mathrm{OD}$ ) of 1-3.

\begin{tabular}{|c|c|c|c|c|c|c|}
\hline \multirow{2}{*}{ Position } & \multicolumn{2}{|c|}{1} & \multicolumn{2}{|c|}{2} & \multicolumn{2}{|c|}{3} \\
\hline & $\delta_{H}$ & $\delta_{\mathrm{C}}$ & $\delta_{H}$ & $\delta_{C}$ & $\delta_{H}$ & $\delta_{C}$ \\
\hline 1 & $2.03,2.73, \mathrm{~m}$ & 33.66 & $1.33,2.70, \mathrm{~m}$ & 31.55 & $\begin{array}{l}\text { 6.94, d, } \\
(12.32)\end{array}$ & 145.70 \\
\hline 2 & $2.30,2.53, \mathrm{~m}$ & 30.90 & $2.19,2.71, \mathrm{~m}$ & 33.18 & $\begin{array}{l}5.82, \mathrm{~d} \\
(12.09)\end{array}$ & 119.11 \\
\hline 3 & & 177.05 & & 176.31 & & 169.08 \\
\hline 4 & & 76.05 & & 76.84 & & 82.15 \\
\hline 5 & $1.46, \mathrm{~m}$ & 51.46 & $1.88, \mathrm{~m}$ & 46.59 & $2.52, \mathrm{~m}$ & 50.01 \\
\hline 6 & $1.21,1.60, \mathrm{~m}$ & 28.38 & $0.72,1.44, \mathrm{~m}$ & 26.44 & $2.15,2.43, \mathrm{~m}$ & 30.73 \\
\hline
\end{tabular}


Table 1. Cont

\begin{tabular}{|c|c|c|c|c|c|c|}
\hline \multirow{2}{*}{ Position } & \multicolumn{2}{|c|}{1} & \multicolumn{2}{|c|}{2} & \multicolumn{2}{|l|}{3} \\
\hline & $\delta_{H}$ & $\delta_{C}$ & $\delta_{H}$ & $\delta_{C}$ & $\delta_{H}$ & $\delta_{C}$ \\
\hline 7 & $1.54,1.74, \mathrm{~m}$ & 27.36 & $1.04,1.29, \mathrm{~m}$ & 27.03 & $2.27,2.58, \mathrm{~m}$ & 39.19 \\
\hline 8 & $2.15, \mathrm{~m}$ & 44.84 & $1.36, \mathrm{~m}$ & 50.24 & & 176.40 \\
\hline 9 & & 146.02 & & 23.88 & & 133.01 \\
\hline 10 & & 45.83 & & 28.01 & & 142.70 \\
\hline 11 & $5.46, \mathrm{~d},(5.85)$ & 118.63 & $1.27,2.21, \mathrm{~m}$ & 27.64 & & 199.85 \\
\hline 12 & $1.98,2.23, \mathrm{~m}$ & 38.96 & $1.72, \mathrm{~m}$ & 34.28 & $2.52,2.83, \mathrm{~m}$ & 49.93 \\
\hline 13 & & 45.29 & & 46.57 & & 48.61 \\
\hline 14 & & 48.13 & & 49.77 & & 54.39 \\
\hline 15 & $1.42,1.45, \mathrm{~m}$ & 34.95 & $1.39, \mathrm{~m}$ & 37.23 & $1.62,1.88, \mathrm{~m}$ & 31.89 \\
\hline 16 & $1.45,1.83, \mathrm{~m}$ & 27.69 & $1.41,1.83, \mathrm{~m}$ & 27.97 & $1.56,1.98, \mathrm{~m}$ & 26.34 \\
\hline 17 & $1.72, \mathrm{~m}$ & 48.01 & $1.69, \mathrm{~m}$ & 49.46 & $1.96, \mathrm{~m}$ & 47.10 \\
\hline 18 & $3 \mathrm{H}, 0.75, \mathrm{~s}$ & 14.93 & $3 \mathrm{H}, 1.05, \mathrm{~s}$ & 18.91 & $3 \mathrm{H}, 0.92, \mathrm{~s}$ & 17.50 \\
\hline 19 & $3 \mathrm{H}, 1.21, \mathrm{~s}$ & 27.77 & $0.55,0.72, \mathrm{~m}$ & 32.40 & $6.72, \mathrm{~s}$ & 138.92 \\
\hline 20 & $1.97, \mathrm{~m}$ & 40.59 & $1.96, \mathrm{~m}$ & 40.64 & $1.99, \mathrm{~m}$ & 40.64 \\
\hline 21 & $\begin{array}{c}3 \mathrm{H}, 1.00, \mathrm{~d}, \\
(6.61)\end{array}$ & 13.49 & $\begin{array}{l}3 \mathrm{H}, 0.98, \mathrm{~d}, \\
\quad(6.49)\end{array}$ & 13.37 & $\begin{array}{l}3 \mathrm{H}, 0.99, \mathrm{~d}, \\
\quad(6.43)\end{array}$ & 13.70 \\
\hline 22 & $\begin{array}{c}4.51, \mathrm{dt} \\
(3.57,13.22)\end{array}$ & 82.24 & $\begin{array}{c}4.51, \mathrm{dt} \\
(3.60,13.24)\end{array}$ & 82.28 & $\begin{array}{c}4.51, \mathrm{dt} \\
(3.53,13.14)\end{array}$ & 81.69 \\
\hline 23 & $2.23,2.40, \mathrm{~m}$ & 24.37 & $2.23,2.40, \mathrm{~m}$ & 24.39 & $2.26,2.38, \mathrm{~m}$ & 24.41 \\
\hline 24 & $6.76, d,(6.47)$ & 142.32 & $6.76, d,(6.47)$ & 142.38 & $6.74, \mathrm{~d},(6.38)$ & 142.18 \\
\hline 25 & & 128.61 & & 128.64 & & 128.66 \\
\hline 26 & & 168.87 & & 168.94 & & 168.70 \\
\hline 27 & $3 \mathrm{H}, 1.87, \mathrm{~s}$ & 16.96 & $3 \mathrm{H}, 1.86, \mathrm{~s}$ & 16.99 & $3 \mathrm{H}, 1,84, \mathrm{~s}$ & 16.95 \\
\hline 28 & $3 \mathrm{H}, 1.27, \mathrm{~s}$ & 28.26 & $3 \mathrm{H}, 1.20, \mathrm{~s}$ & 26.23 & $3 \mathrm{H}, 1.50, \mathrm{~s}$ & 26.28 \\
\hline 29 & $3 \mathrm{H}, 1.28, \mathrm{~s}$ & 33.44 & $3 \mathrm{H}, 1.21, \mathrm{~s}$ & 31.30 & $3 \mathrm{H}, 1.37, \mathrm{~s}$ & 29.11 \\
\hline 30 & $3 \mathrm{H}, 0.79, \mathrm{~s}$ & 18.81 & $3 \mathrm{H}, 0.98, \mathrm{~s}$ & 20.09 & $3 \mathrm{H}, 1.32, \mathrm{~s}$ & 27.25 \\
\hline 31 & $4.11, \mathrm{q},(7.13)$ & 61.40 & $4.08, \mathrm{q},(7.12)$ & 61.39 & & \\
\hline 32 & $\begin{array}{c}3 \mathrm{H}, 1.25, \mathrm{t} \\
\quad(7.14)\end{array}$ & 14.57 & $\begin{array}{c}3 \mathrm{H}, 1.22, \mathrm{t} \\
(7.15)\end{array}$ & 14.58 & & \\
\hline
\end{tabular}

The ethyl esters of compounds 1-2 may have been obtained during the extraction with ethanol. The compounds 1-7 were tested for cytotoxic effects against the Hep-G2 human tumor cell lines. Compared with the positive control cisplatin $\left(\mathrm{IC}_{50}=3.16 \mu \mathrm{g} / \mathrm{mL}\right)$, compounds 1-7 displayed no apparent cytotoxicity $\left(\mathrm{IC}_{50}>50 \mu \mathrm{g} / \mathrm{mL}\right.$ ).

\section{Materials and Methods}

\subsection{General}

Optical rotation was recorded on Autopol IV Automatic Polarimeter (RUDOLPH, Hackettstown, NJ, USA). IR spectra (Figures S10, S19 and S27) were obtained using the IRT racer-100 (SHIMADZU, Kyoto, Japan) with KBr pellets. NMR spectra were recorded on a Bruker DXR-600 instrument (600 MHz for ${ }^{1} \mathrm{H}$ and $150 \mathrm{MHz}$ for ${ }^{13} \mathrm{C}$ ) with TMS as an internal standard, and the deuterated solvent $\left(\mathrm{CD}_{3} \mathrm{OD}\right)$ were used to solubilize the samples. HR-ESI-MS was recorded on a UPLC-Q Exactive MS system (Thermo Fisher, Santa Clara, CA, USA). Silica gel (200-300, 300-400 mesh, Qingdao Haiyang Chemical Co., Ltd., Qingdao, China) was used for the chromatography column. Semi-preparative HPLC was performed on an Agilent Technologies 1260 Infinity II system equipped with a diode array detector and C18 column ( $250 \mathrm{~mm} \times 9.6 \mathrm{~mm}, 5 \mu \mathrm{m}$, Agilent Technologies, Santa Clara, CA, USA).

\subsection{Plant Material}

The plant of S. chinensis (Turcz) Baill. Was collected in 2009 from Shennongjia County, Hubei province, China and identified by Prof. Mao-Chuan Liao from the School of Pharmaceutical Sciences 
at South-Central University for Nationalities, China. The voucher specimen (No. 2009050301) was deposited in the Herbarium of the School of Pharmaceutical Sciences, South-Central University for Nationalities, China.

\subsection{Extraction and Isolation}

The dried plant material of S. chinensis (Turcz) Baill. $(10 \mathrm{Kg}$ ) was extracted four times with $95 \%$ ethanol $(25 \mathrm{~L})$ at room temperature, 48 hours each time. After concentration, the extract was suspended in hot water and then partitioned with petroleum ether and EtOAc to give petroleum ether (A) and EtOAc ether (B, $151 \mathrm{~g})$ fractions, respectively. Fraction B was chromatographed over silica gel (2 Kg), eluting with EtOAc in petroleum ether (0-100\%, stepwise), yielding six fractions (Fr1-Fr6). Fraction Fr1 (2.71 g) was chromatographed over ODS MPLC eluting with $\mathrm{H}_{2} \mathrm{O}-\mathrm{MeOH}(0-100 \%$, stepwise), yielding 40 fractions (Fr1-1-Fr1-40). Fr1-39 was further purified by semi-preparative HPLC using $\mathrm{MeOH}-\mathrm{H}_{2} \mathrm{O}(90: 10, v / v)$ to provide compound $\mathbf{1}(6.8 \mathrm{mg}, \mathrm{Rt}=14.8 \mathrm{~min})$. Fr1-40 was further purified by semi-preparative $\mathrm{HPLC}$ using $\mathrm{MeOH}-\mathrm{H}_{2} \mathrm{O}(88: 12, v / v)$ to provide compound 2 (3.6 $\left.\mathrm{mg}, \mathrm{Rt}=16.9 \mathrm{~min}\right)$. Using the same ways, Fr3-29 was further purified by semi-preparative HPLC using $\mathrm{MeCN}^{-\mathrm{H}_{2} \mathrm{O}}$ (45:55-60:40, $20 \mathrm{~min}, v / v$ ) to provide compound 3 ( $2.7 \mathrm{mg}$, $\mathrm{Rt}=18.8 \mathrm{~min}$ ). Fr3-35 was further purified

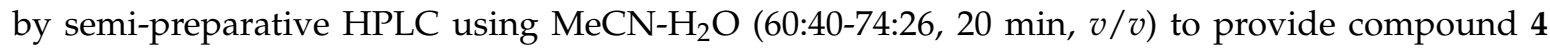
( $4.5 \mathrm{mg}, \mathrm{Rt}=12.2 \mathrm{~min}$ ) and compound $5(1.6 \mathrm{mg}, \mathrm{Rt}=13.0 \mathrm{~min})$. Fr3-26 was further purified by semi-preparative HPLC using $\mathrm{MeOH}-\mathrm{H}_{2} \mathrm{O}(62: 38, v / v)$ to provide compound 6 ( $2.1 \mathrm{mg}$, $\left.\mathrm{Rt}=16.8 \mathrm{~min}\right)$. Fr3-30 was further purified by semi-preparative HPLC using $\mathrm{MeCN}_{-} \mathrm{H}_{2} \mathrm{O}(50.5: 49.5, v / v)$ to provide compound 7 ( $1.5 \mathrm{mg}$, $\mathrm{Rt}=36.2 \mathrm{~min})$.

\subsection{MTT Assay for Measuring Cell Cytotoxicity}

Cytotoxicity assay was performed against human Hep-G2 tumor cell lines, which were seeded in 96-well plates and incubated for $24 \mathrm{~h}$. The cells were incubated in DMEM medium. Subsequently, the cells were treated with concentration gradient of compounds 1-7 (cisplatin was employed as positive control) and incubated at $37^{\circ} \mathrm{C}$ in $5 \% \mathrm{CO}_{2}$ for $24 \mathrm{~h}$. Then, $10 \mu \mathrm{L}$ MTT was added to each well and the mixture was incubated for $5 \mathrm{~h}$. The medium was removed and after adding DMSO ( $150 \mu \mathrm{L} /$ well) to each plate, the formazan crystals were dissolved and then absorbance was determined with microplate a reader at $490 \mathrm{~nm}$.

\subsection{Experimental Data of Identified Compounds}

Compound Schisanlactone I (1): White amorphous powder. $[\alpha]_{\mathrm{D}}^{25}=+76^{\circ}(\mathrm{c}=0.07, \mathrm{MeOH}), \mathrm{IR}(\mathrm{KBr})$ $v_{\max } 3455,3445,3441,3435,2947,2357,1651,1227 \mathrm{~cm}^{-1}$, HR-ESI-MS: $m / z 515.3737[\mathrm{M}+\mathrm{H}]^{+}$(calculated for $\left.\mathrm{C}_{32} \mathrm{H}_{51} \mathrm{O}_{5}, 515.3758\right),{ }^{1} \mathrm{H}-\mathrm{NMR}\left(\mathrm{CD}_{3} \mathrm{OD}, 600 \mathrm{MHz}\right)$ and ${ }^{13} \mathrm{C}-\mathrm{NMR}\left(\mathrm{CD}_{3} \mathrm{OD}, 150 \mathrm{MHz}\right)$ (see Table 1$)$.

X-ray crystal structure analysis compound 1 : $\mathrm{C}_{32} \mathrm{H}_{50} \mathrm{O}_{5}(\mathrm{M}=514.72)$ monoclinic, space group, $P 12{ }_{1} 1$, $\mathrm{Z}=2, a=9.2604(2) \AA, b=11.0694(3) \AA, c=14.7395(4) \AA, \alpha=\gamma=90^{\circ}, \beta=92.2800(10)^{\circ}, \mathrm{V}=1509.71(7) \AA^{3}$. $\mu(\mathrm{Cu} \mathrm{K} \alpha)=0.587 \mathrm{~mm}^{-1}, D_{\text {calc }}=1.132 \mathrm{~g} / \mathrm{cm}^{3} ; \mathrm{S}=1.039$, final $R_{1}=0.0454, \mathrm{w} R_{2}=0.1289$ for 5583 observed from 6124 independent and 34,321 measured reflections $\left(\theta_{\max }=74.56, \mathrm{I}>2 \sigma(\mathrm{I})\right.$, criterion and 346 parameters), maximum and minimum residues are 0.303 and $-0.126 \mathrm{e}^{-3}$. The Flack parameter value was 0.04 (9), indicating that the absolute configuration of 1 was determined to be $(5 R, 8 S, 10 S$, $13 R, 14 S, 17 R, 20 S, 22 R$ ) correctly. Crystallographic data of 1 have been deposited in the Cambridge Crystallographic data Center (no. CCDC 1847542).

Compound Schinalactone D (2): White amorphous powder. $[\alpha]_{\mathrm{D}}^{25}=+75^{\circ}(\mathrm{c}=0.05, \mathrm{MeOH}), \mathrm{IR}(\mathrm{KBr})$ $v_{\max } 3479,3458,3452,3444,3429,3417,1645,1259,1222 \mathrm{~cm}^{-1}$, HR-ESI-MS: $m / z 537.3551\left[\mathrm{M}+\mathrm{Na}^{+}\right.$ (calculated for $\left.\mathrm{C}_{32} \mathrm{H}_{50} \mathrm{O}_{5} \mathrm{Na}, 537.3556\right),{ }^{1} \mathrm{H}-\mathrm{NMR}\left(\mathrm{CD}_{3} \mathrm{OD}, 600 \mathrm{MHz}\right)$ and ${ }^{13} \mathrm{C}-\mathrm{NMR}\left(\mathrm{CD}_{3} \mathrm{OD}, 150 \mathrm{MHz}\right)$ (see Table 1). 
Compound Schisanlactone J (3): White amorphous powder. $[\alpha]_{\mathrm{D}}^{25}=+119^{\circ}(\mathrm{c}=0.03, \mathrm{MeOH}), \mathrm{IR}(\mathrm{KBr})$ $v_{\max } 3462,3448,3442,3421,2083,1637,1257 \mathrm{~cm}^{-1}$, HR-ESI-MS: $m / z 479.2790[\mathrm{M}+\mathrm{H}]^{+}$(calculated for $\left.\mathrm{C}_{30} \mathrm{H}_{39} \mathrm{O}_{5}, 479.2798\right),{ }^{1} \mathrm{H}-\mathrm{NMR}\left(\mathrm{CD}_{3} \mathrm{OD}, 600 \mathrm{MHz}\right)$ and ${ }^{13} \mathrm{C}-\mathrm{NMR}\left(\mathrm{CD}_{3} \mathrm{OD}, 150 \mathrm{MHz}\right)$ (see Table 1$)$.

\section{Conclusions}

Three new triterpenoids and four known triterpenoids were isolated from the S. chinensis (Turcz) Baill. The seven compounds were tested for cytotoxic effects against the Hep-G2 human tumor cell lines, which displayed no apparent cytotoxicity.

Supplementary Materials: The following are available online, Figure S1. ${ }^{1} \mathrm{H}-\mathrm{NMR}\left(600 \mathrm{MHz}, \mathrm{CD}_{3} \mathrm{OD}\right)$ spectrum of compound 1, Figure S2. ${ }^{13} \mathrm{C}-\mathrm{NMR}\left(150 \mathrm{MHz}, \mathrm{CD}_{3} \mathrm{OD}\right)$ spectrum of compound 1, Figure S3. DEPT $135^{\circ}$ spectrum of compound 1, Figure S4. ${ }^{1} \mathrm{H}^{-1} \mathrm{H}$ COSY spectrum of compound 1, Figure S5. HSQC spectrum of compound 1, Figure S6. HMBC spectrum of compound 1, Figure S7. Key HMBC correlations and ${ }^{1} \mathrm{H}^{-1} \mathrm{H}$ COSY correlations of compound 1, Figure S8. X-ray SAINT drawing of compound 1, Figure S9. HR-ESI-MS spectrum of compound 1, Figure S10. IR spectrum of compound 1, Figure S11. ${ }^{1} \mathrm{H}-\mathrm{NMR}\left(600 \mathrm{MHz}, \mathrm{CD}_{3} \mathrm{OD}\right)$ spectrum of compound 2, Figure S12. ${ }^{13} \mathrm{C}-\mathrm{NMR}\left(150 \mathrm{MHz}, \mathrm{CD}_{3} \mathrm{OD}\right)$ spectrum of compound 2, Figure S13. DEPT $135^{\circ}$ spectrum of compound 2, Figure S14. ${ }^{1} \mathrm{H}-{ }^{1} \mathrm{H}$ COSY spectrum of compound 2, Figure S15. HSQC spectrum of compound 2, Figure S16. HMBC spectrum of compound 2, Figure S17. Key HMBC correlations and ${ }^{1} \mathrm{H}^{-1} \mathrm{H}$ COSY correlations of compound 2, Figure S18. HR-ESI-MS spectrum of compound 2, Figure S19. IR spectrum of compound 2, Figure S20. ${ }^{1} \mathrm{H}-\mathrm{NMR}\left(600 \mathrm{MHz}, \mathrm{CD}_{3} \mathrm{OD}\right)$ spectrum of compound 3, Figure S21. ${ }^{13} \mathrm{C}-\mathrm{NMR}\left(150 \mathrm{MHz}, \mathrm{CD}_{3} \mathrm{OD}\right)$ spectrum of compound 3, Figure S22. DEPT $135^{\circ}$ spectrum of compound 3, Figure S23. ${ }^{1} \mathrm{H}^{-}{ }^{1} \mathrm{H}$ COSY spectrum of compound 3, Figure S24. HSQC spectrum of compound 3, Figure S25. HMBC spectrum of compound 3, Figure S26. HR-ESI-MS spectrum of compound 3, Figure S27. IR spectrum of compound 3.

Author Contributions: X.-X.L. and F.Q. conceived designed the experiments. F.Q., H.L., P.C. and S.-J.L. performed the experiments. F.Q. analyzed the data. H.D. and G.-Z.Y. contributed regents, materials, analysis tools. F.Q. and X.-X.L. wrote the paper.

Funding: This research was funded by the Natural Science Foundation of China (No. 21572164), Sino-German Center for Research Promotion (GZ1289), Key Projects of Technological Innovation of Hubei Province (No. 2016ACA138).

Acknowledgments: This work was supported by the Natural Science Foundation of China (No. 21572164), Sino-German Center for Research Promotion (GZ1289), Key Projects of Technological Innovation of Hubei Province (No. 2016ACA138).

Conflicts of Interest: The authors declare no conflict interest.

\section{References}

1. Huang, S.X.; Li, R.T.; Liu, J.P.; Lu, Y.; Chang, Y.; Lei, C.; Xiao, W.L.; Yang, L.B.; Zheng, Q.T.; Sun, H.D. Isolation and characterization of biogenetically related highly oxygenated nortriterpenoids from Schisandra chinensis. Org. Lett. 2007, 9, 2079-2082. [CrossRef] [PubMed]

2. Szopa, A.; Ekiert, R.; Ekiert, H. Current knowledge of Schisandra chinensis (Turcz.) Baill. (Chinese magnolia vine) as a medicinal plant species: A review on the bioactive components, pharmacological properties, analytical and biotechnological studies. Phytochem. Rev. 2017, 16, 195-218. [CrossRef] [PubMed]

3. Szopa, A.; Klimek-Szczykutowicz, M.; Kokotkiewicz, A.; Maslanka, A.; Krol, A.; Luczkiewicz, M.; Ekiert, H. Phytochemical and biotechnological studies on Schisandra chinensis cultivar Sadova No. 1-a high utility medicinal plant. Appl. Microbiol. Biotechnol. 2018, 102, 5105-5120. [CrossRef] [PubMed]

4. Hancke, J.L.; Burgos, R.A.; Ahumada, F. Schisandra chinensis (Turcz.) Baill. Fitoterapia 1999, 70, 451-471. [CrossRef]

5. Song, J.; Zhou, M.; Zhou, J.; Liang, J.J.; Peng, X.G.; Liu, J.; Ruan, H.L. Schincalactones A and B, Two 5/5/6/11/3 Fused Schinortriterpenoids with a 13-Membered Carbon Ring System from Schisandra incarnata. Org. Lett. 2018, 20, 2499-2502. [CrossRef] [PubMed]

6. Xiao, W.L.; Yang, L.M.; Gong, N.B.; Wu, L.; Wang, R.R.; Pu, J.X.; Li, X.L.; Huang, S.X.; Zheng, Y.T.; Li, R.T.; et al. Rubriflordilactones A and B, two novel bisnortriterpenoids from Schisandra rubriflora and their biological activities. Org. Lett. 2006, 8, 991-994. [CrossRef] [PubMed]

7. Panossian, A.; Wikman, G. Pharmacology of Schisandra chinensis Bail: An overview of Russian research and uses in medicine. J. Ethnopharmacol. 2008, 118, 183-212. [CrossRef] [PubMed] 
8. Guo, L.Y.; Hung, T.M.; Bae, K.H.; Shin, E.M.; Zhou, H.Y.; Hong, Y.N.; Kang, S.S.; Kim, H.P.; Kim, Y.S. Anti-inflammatory effects of schisandrin isolated from the fruit of Schisandra chinensis Baill. Eur. J. Pharmacol. 2008, 591, 293-299. [CrossRef] [PubMed]

9. Jiang, Y.; Yang, G.Z.; Chen, Y.; Liao, M.C.; Liu, X.M.; Chen, S.; Liu, L.; Lei, X.X. Terpenes from Schisandra sphenanthera. Helv. Chim. Acta 2011, 94, 491-496. [CrossRef]

10. Shen, Y.C.; Lin, Y.C.; Chiang, M.Y.; Yeh, S.F.; Cheng, Y.B.; Liao, C.C. Kadsuphilactones A and B, two new triterpene dilactones from kadsuraphilippinensis. Org. Lett. 2005, 7, 3307-3310. [CrossRef] [PubMed]

11. Liu, J.S.; Huang, M.F. On the structures of schisanlactone $C$ and schisanlactone D, two new triterpene lactones from Schisandra sp. Acta Chim. Sin. 1984, 42, 464-469.

12. Song, Q.Y.; Jiang, K.; Zhao, Q.Q.; Gao, K.; Jin, X.J.; Yao, X.J. Eleven new highly oxygenated triterpenoids from the leaves and stems of Schisandra chinensis. Org. Biomol. Chem. 2013, 11, 1251-1258. [CrossRef] [PubMed]

13. Zhou, S.Y.; Wang, W.G.; Li, H.M.; Zhang, R.B.; Li, H.Z.; Li, R.T. Schisanlactone H and sphenanthin A, new metabolites from Schisandra sphenanthera. J. Asian Nat. Prod. Res. 2009, 11, 861-866. [CrossRef] [PubMed]

14. He, F.; Pu, J.X.; Huang, S.X.; Wang, Y.Y.; Xiao, W.L.; Li, L.M.; Liu, J.P.; Zhang, H.B.; Li, Y.; Sun, H.D. Schinalactone A, a new cytotoxic triterpenoid from Schisandra sphenanthera. Org. Lett. 2010, 12, 1208-1211. [CrossRef] [PubMed]

15. Chen, D.F.; Zhang, S.X.; Wang, H.K.; Zhang, S.Y.; Sun, Q.Z.; Cosentino, L.M.; Lee, K.H. Novel anti-HIV lancilactone $C$ and related triterpenes from Kadsura lancilimba. J. Nat. Prod. 1999, 62, 94-97. [CrossRef] [PubMed]

Sample Availability: Samples of the compounds are not available from the authors.

(C) 2018 by the authors. Licensee MDPI, Basel, Switzerland. This article is an open access article distributed under the terms and conditions of the Creative Commons Attribution (CC BY) license (http:// creativecommons.org/licenses/by/4.0/). 